\title{
FDA perspective on anti-TNF treatments
}

\author{
William D Schwieterman
}

Although there are many points I would like to make about the FDA's perspective on antiTNF treatments, I have limited myself to those 10 that I believe are most important. I present several of these less as absolutes than as stimulants for discussion, as I wish to make use of the expertise at this conference for furthering our collective understanding of how anti-TNF agents might best be used.

1 The long term safety of anti-TNF agents is unclear

There are no long term data on the safety of anti-TNF agents. Controlled safety data exist, to date, only from studies of durations of six months or less. Given that TNF is known to be a potent immunomodulatory agent, questions exist about the safety of its long term inhibition with anti-TNF agents, particularly with regard to the treated patient's immunological defence against infections. In addition, some have questioned whether a higher incidence of malignancies are associated with use of some anti-TNF agents. More studies, including registries and controlled trials of longer durations, may be helpful for answering questions in this area. Until more safety data are available, the rheumatology community is correct to recommend reserving the use of anti-TNF agents only for patients for which it is clearly indicated.

\section{The long term efficacy of anti-TNF agents is unclear}

Just as there are questions about the long term safety of anti-TNF agents, there are questions about their long term efficacy. As mentioned for safety above, no controlled efficacy data have been generated, to date, for treatment regimens longer than six months. Although it is thought likely by some, it is by no means certain that there exists a correlation between symptomatic and structural benefits after treatment with anti-TNF agents. The FDA encourages (in the RA Guidance Document) sponsors to study anti-TNF agents for the treatment of RA for at least one year to adequately characterise their long term safety and efficacy. These long term studies are particularly important for measuring structural, functional and quality of life measures.

Immunology and Infectious Disease Branch, Division of Clinical Trial Design and Analysis, Food and Drug Administration, 1401 Rockville Pike, Rockville, MD 20852, USA

Correspondence to: Dr W D Schwieterman. many experimental systems to have different binding avidities for TNF, different immunogenicity profiles, different pharmacokinetic profiles, etc. Antibodies directed against TNF can act much differently than molecules generated from cell-surface receptors. It is also well known that anti-TNF agents have different effects in different diseases. For example, etanercept (TNFR:Fc) both increases the mortality of patients with sepsis and reduces symptoms in patients with RA. Sponsors need to be cognisant of the differences between antiTNF agents when submitting proposals to the agency.

\section{New products need not beat Enbrel for approval}

The agency has the legal mandate to approve products that are safe and effective, and the legal mandate to protect the public from harm. All clinical trials, therefore, need have equipoise and incorporate provisions for use of the current standard of care. Although Enbrel has been shown to be safe and efficacious, it has not yet been established as the standard of care in all populations with RA. Various RA subpopulations, including those with early or moderate disease, might ethically be studied without an Enbrel comparator arm. Other protocols in patients with severe disease might also be possible without an Enbrel comparator arm if (a) data from early trials support efficacy, and if (b) appropriate stopping rules forbidding the long term treatment of patients failing treatment (also known as "early escape" rules) are incorporated into them.

\section{The ACR 20 alone is not adequate for some purposes}

While there are many good reasons to use the ACR 20 as an outcome measure, and I both congratulate and thank the many who worked hard to generate this widely used index, the ACR 20 is not useful for all purposes. A discussion of this is particularly important now that several potent new treatments are being developed and have been marketed for the treatment of RA. The ACR 20 is a generally poor discriminator of efficacy, given that it is a categorical outcome measure set at a relatively low threshold. Powerful new treatments almost frequently induce at least a $20 \%$ reduction in signs and symptoms for the patients they benefit. As larger clinical effects are impossible to measure with the ACR 20 alone, and as dose effects are difficult to discern with the ACR 20, other "continuous" outcomes should also be considered in studies of RA. These include "area under the curve" measurements, tender and swollen joint counts, etc. The Smirnov or other statistical tests, which uses groups (for 
example, ACR 20-50; 50-70; 70-100) or ranks of outcomes to full advantage can also be quite useful to discriminate various parameters of efficacy.

\section{Unblinding effects are often problematic}

The sometimes dramatic and often rapid onset of clinical effects after treatment with antiTNF agents can sometimes be a disadvantage for clinical trialists, as these effects may lead to unblinding of the patient and/or investigator. In addition, other effects, including adverse effects, have been observed with anti-TNF agents and might also lead to unblinding. Because many of the components of the ACR efficacy indices (that is, ACR 20,50, 70) are subjective, and because the placebo effect in studies of RA is known to be high, great care must be taken by investigators to ensure that bias from unblinding is not introduced into studies of anti-TNF agents. Patient questionnaires are sometimes helpful, as are third party blinded evaluators of primary outcome measures. The agency strongly recommends that, when questions about bias introduced by blinding exist (as they frequently do for anti-TNF agents), independent third party blinded evaluators be used to assess primary outcome measures.

\section{Long term trials in which standard of care is denied are unethical}

There has been much debate recently about the propriety of studies using patients who fail to receive standard of care for long periods of time. The agency believes that, (a) given the number of effective agents now available to RA patients and, (b) given the increasing evidence that these agents prevent the structural and irreversible morbidities that otherwise manifest in patients with active disease, no trial longer than six months should have an arm in which standard of care (for example, DMARDS) is denied. It is important to recognise that giving placebo and denying standard of care are not the same. Placebos are often given in addition to standard of care and, when given as such, may be given ethically for any amount of time

\section{Study of broad patient populations is important}

Although there often is merit in early product development to studying a relatively homogeneous patient population, the agency almost always asks sponsors to study in later development a broad range of patient types. Because the risks and benefits of treatments often differ in different patient subgroups, and because it is important that the package insert for any approved product provide meaningful information on these subgroups, phase 3 trials should be designed to incorporate a broad, heterogeneous patient population. This is true even if the expectation exists that a product may be effective, or more effective, in a particular subgroup, as "evaluable" groups can be used in the primary efficacy analysis to minimise type 2 error (false negative). Finally, the agency generally discourages study patients with very early or very late stage disease in initial trials of new products.

\section{Dose ranging and dose optimisation is important}

Characterising the optimal dose of a therapeutic agent is one of the more difficult tasks of product development, but one that is important. Although Enbrel is an effective treatment for RA in many patients, no plateau for efficacy was observed in one six month trial. Given that $30-40 \%$ of patients appear not to respond to Enbrel, given the absence of a clear efficacy plateau, and given the presence of other data suggesting a linear relation between dose and clinical benefit, there exist questions about how best to treat patients unresponsive or partially responsive to this agent. Similar questions exist for other agents. Immunex, to their credit, is pursuing studies of higher doses of Enbrel to further evaluate their product's optimal dose. Other sponsors of anti-TNF treatments should also work to define a maximally tolerated dose and to optimise other aspects of a dosing regimen when submitting proposals to the agency. Phase 2 studies are often useful in this regard.

\section{Anti-TNF agents for RA constitute a} major therapeutic advance

I want to end on a positive note because I feel positive about this area and this field. Only recently was the RA community struggling to devise sensitive outcome measures for the testing of agents with marginal efficacy. Now, new agents are available, and more powerful ones are being developed, which bring hope to patients and investigators alike. Many promise to ameliorate, or even prevent, the long term structural damage and physical dysfunction associated with RA. I believe we have entered a new era, and that despite the many unanswered questions, we should be optimistic about our chances for effectively fighting this common and often debilitating disease. 